S. BELMEHDI (Lille)

S. LEW ANOWICZ (Wrocław)

A. RONVEAUX (Namur)

\title{
LINEARIZATION OF THE PRODUCT OF ORTHOGONAL POLYNOMIALS OF A DISCRETE VARIABLE
}

Abstract. Let $\left\{P_{k}\right\}$ be any sequence of classical orthogonal polynomials of a discrete variable. We give explicitly a recurrence relation (in $k$ ) for the coefficients in $P_{i} P_{j}=\sum_{k} c(i, j, k) P_{k}$, in terms of the coefficients $\sigma$ and $\tau$ of the Pearson equation satisfied by the weight function $\varrho$, and the coefficients of the three-term recurrence relation and of two structure relations obeyed by $\left\{P_{k}\right\}$.

1. Introduction. Let $\left\{P_{k}(x)\right\}$ be any system of classical orthogonal polynomials of a discrete variable, i.e., Charlier polynomials $C_{k}(x ; a)$, Meixner polynomials $M_{k}(x ; \beta, c)$, Krawtchouk polynomials $K_{k}(x ; p, N)$, or Hahn polynomials $Q_{n}(x ; \alpha, \beta, N)$ :

$$
\sum_{x=0}^{B-1} \varrho(x) P_{k}(x) P_{l}(x)=\delta_{k l} h_{k} \quad(k, l=0,1, \ldots),
$$

where $h_{k}>0(k=0,1, \ldots)$; the set of orthogonality is $\{0,1, \ldots, B-1\}$, where $B$ equals $+\infty,+\infty, N+1$ and $N$, respectively.

Askey and Gasper [2] have given explicit forms for the coefficients in

(1.1) $P_{i}(x) P_{j}(x)=\sum_{k=|i-j|}^{\min (i+j, B-1)} c_{k}^{i j} P_{k}(x) \quad(i, j \geq 0 ; x \in\{0,1, \ldots, B-1\})$,

called the linearization coefficients of the polynomials $\left\{P_{k}\right\}$ (see [1], Lecture 5), in terms of finite or infinite series.

1991 Mathematics Subject Classification: Primary 33C45, 33E30.

Key words and phrases: linearization coefficients; classical orthogonal polynomials of a discrete variable; recurrence relations. 
The aim of this paper is to show that $c_{k}^{i j}$ obey a linear recurrence relation

$$
\mathcal{L}^{*} c_{k}^{i j} \equiv \sum_{h=0}^{r} A_{h}^{*}(k) c_{k+h}^{i j}=0 .
$$

Recurrence (1.2) may serve as a basis for a very efficient backward recursion algorithm for evaluating these coefficients. The difference operator $\mathcal{L}^{*}$ is given explicitly in terms of the coefficients $\sigma$ and $\tau$ of the Pearson equation (see (2.2) below) satisfied by the weight function $\varrho$, and the coefficients of the three-term recurrence relation (see (2.1)) and of structure relations obeyed by $\left\{P_{k}\right\}$ (see $(2.5),(2.6)$ ). This result is contained in Theorem 3.5; applications to some systems of polynomials are given.

The main tool used in the derivation of the recurrence relation is the fourth-order difference equation

$$
\boldsymbol{Q}_{4} w=0
$$

obeyed by the product $w:=P_{i} P_{j}$. We give a determinantal form (see Theorem 3.1), as well as two (equivalent) almost factorized forms of the fourth-order operator $\boldsymbol{Q}_{4}$ (see Corollary 3.2 and Theorem 3.4).

\section{Properties of the classical orthogonal polynomials}

2.1. Basics of classical orthogonal polynomials of a discrete variable. In the sequel, we make use of certain properties enjoyed by all classical families of orthogonal polynomials (see [4], Chapter VI; [5]; [6]; [9], Chapter II; or [10]). Besides the three-term recurrence relation

$$
\begin{aligned}
x P_{k}(x)=\xi_{0}(k) P_{k-1}(x)+ & \xi_{1}(k) P_{k}(x)+\xi_{2}(k) P_{k+1}(x) \\
& \left(k=0,1, \ldots ; P_{-1}(x) \equiv 0, P_{0}(x) \equiv 1\right)
\end{aligned}
$$

we need four other properties.

First, the weight function $\varrho$ satisfies a difference equation of the Pearson type

$$
\boldsymbol{\Delta}[\sigma(x) \varrho(x)]=\tau(x) \varrho(x),
$$

where $\sigma$ is a polynomial of degree at most 2, and $\tau$ is a first-degree polynomial.

Second, for arbitrary $i$, the polynomial $P_{i}$ obeys the second order difference equation

$$
\boldsymbol{P}_{2}^{(n)} P_{i}(x) \equiv\left\{\sigma(x) \boldsymbol{\Delta} \boldsymbol{\nabla}+\tau(x) \boldsymbol{\Delta}+\lambda_{i} \boldsymbol{I}\right\} P_{i}(x)=0,
$$

where $\boldsymbol{\Delta}:=\boldsymbol{E}-\boldsymbol{I}, \boldsymbol{\nabla}:=\boldsymbol{I}-\boldsymbol{E}^{-1}, \boldsymbol{E}^{m}(m \in \mathbb{Z})$ is the $m$ th shift operator, $\boldsymbol{E}^{m} f(x)=f(x+m), \boldsymbol{I}$ is the identity operator, $\boldsymbol{I} f(x)=f(x)$, and $\lambda_{i}$ is the constant given by

$$
\lambda_{i}:=-\frac{1}{2} i\left[(i-1) \sigma^{\prime \prime}+2 \tau^{\prime}\right] \quad(i \in \mathbb{N}) .
$$


(By convention, all the bold letter operators act on the variable $x$.)

Third, we have a pair of the so-called structure relations,

$(2.5)[\sigma(x)+\tau(x)] \boldsymbol{\Delta} P_{k}(x)=d_{0}(k) P_{k-1}(x)+d_{1}(k) P_{k}(x)+d_{2}(k) P_{k+1}(x)$,

and

(2.6) $\sigma(x) \nabla P_{k}(x)=d_{0}(k) P_{k-1}(x)+\left[d_{1}(k)+\lambda_{k}\right] P_{k}(x)+d_{2}(k) P_{k+1}(x)$.

Fourth,

$$
\left.\sigma(x) \varrho(x) x^{k}\right|_{x=0} ^{x=B}=0 \quad(k=0,1, \ldots) .
$$

2.2. Identities involving the discrete Fourier coefficients. We shall need certain properties of the Fourier coefficients of an arbitrary polynomial $f$, $\operatorname{deg} f<B$, defined by

$$
a_{k}[f]:=\frac{1}{h_{k}} b_{k}[f] \quad(k=0,1, \ldots, B-1),
$$

where

$$
b_{k}[f]:=\sum_{x=0}^{B-1} \varrho(x) P_{k}(x) f(x)
$$

i.e., the coefficients in the expansion

$$
f=\sum_{k=0}^{\operatorname{deg} f} a_{k}[f] P_{k} .
$$

Let $X, \mathcal{D}$ and $\widetilde{\mathcal{D}}$ be the difference operators (acting on $k$ ) defined by

$$
\begin{aligned}
& \mathcal{X}:=\xi_{0}(k) \mathcal{E}^{-1}+\xi_{1}(k) \mathcal{J}+\xi_{2}(k) \mathcal{E}, \\
& \mathcal{D}:=d_{0}(k) \mathcal{E}^{-1}+d_{1}(k) \mathcal{J}+d_{2}(k) \mathcal{E}, \\
& \widetilde{\mathcal{D}}:=\mathcal{D}+\lambda_{k} \mathcal{J}
\end{aligned}
$$

(cf. (2.1), (2.5) and (2.6), respectively) where $\mathcal{J}$ is the identity operator, and $\mathcal{E}^{m}$ the $m$ th shift operator: $\mathcal{J} b_{k}[f]=b_{k}[f], \mathcal{E}^{m} b_{k}[f]=b_{k+m}[f](m \in \mathbb{Z})$. For the sake of simplicity, we write $\mathcal{E}$ in place of $\mathcal{E}^{1}$. (We adopt the convention that all the script letter operators act on the variable $k$.)

Further, define the difference operators $\boldsymbol{U}, \boldsymbol{V}$ and $\boldsymbol{L}$ (acting on $x$ ) by

$$
\begin{aligned}
\boldsymbol{U} & :=\sigma(x) \boldsymbol{\nabla}+\tau(x) \boldsymbol{I}, \\
\boldsymbol{V} & :=[\sigma(x)+\tau(x)] \boldsymbol{\Delta}+\tau(x) \boldsymbol{I}, \\
\boldsymbol{L} & :=\boldsymbol{V}-\boldsymbol{U},
\end{aligned}
$$

respectively. Notice that since $\Delta \nabla=\boldsymbol{\Delta}-\boldsymbol{\nabla}$, we can write

$$
\boldsymbol{P}_{2}^{(n)}=\boldsymbol{L}+\lambda_{i} \boldsymbol{I} .
$$

Using (2.1)-(2.7), the following lemma can be proved. 
Lemma $2.1([8])$. The coefficients (2.9) obey the identities:

$$
\begin{gathered}
b_{k}[q f]=q(X) b_{k}[f] \quad(q \text { an arbitrary polynomial), } \\
b_{k}[\boldsymbol{U} f]=-\mathcal{D} b_{k}[f], \quad \widetilde{\mathcal{D}} b_{k}[\boldsymbol{\nabla} f]=\lambda_{k} b_{k}[f], \\
b_{k}[\boldsymbol{V} f]=-\widetilde{\mathcal{D}} b_{k}[f], \quad \mathcal{D} b_{k}[\boldsymbol{\Delta} f]=\lambda_{k} b_{k}[f], \\
b_{k}[\boldsymbol{L} f]=-\lambda_{k} b_{k}[f] .
\end{gathered}
$$

\section{Main result}

3.1. Fourth-order difference equation for the product $P_{i} P_{j}$. Using definitions (2.13) and (2.14), equation (2.3) can be written in the following equivalent form:

$$
A(x) y(x+1)+B_{n}(x) y(x)+C(x) y(x-1)=0,
$$

with $y=P_{n}$, and

$$
A:=\sigma+\tau, \quad B_{n}:=\lambda_{n}-2 \sigma-\tau, \quad C:=\sigma .
$$

In the sequel, we adopt the notation

$$
f^{(m)}(x):=\boldsymbol{E}^{m} f(x)=f(x+m) \quad(m \in \mathbb{Z}) .
$$

The following theorem is a slightly improved version of a result of [7].

Theorem 3.1. The product $w:=P_{i} P_{j}(i, j \geq 0, i \neq j)$ satisfies the following difference equation of the fourth order:

$$
\boldsymbol{Q}_{4} w \equiv\left|\begin{array}{ccc}
C^{(1)} C^{(2)} \boldsymbol{R}_{2} w & B_{i} & 1 \\
C^{(2)} \boldsymbol{R}_{3} w & -B_{j}^{(1)} & 1 \\
\boldsymbol{R}_{4} w & B_{i}^{(2)} & 1
\end{array}\right|=0,
$$

where

$$
\begin{aligned}
& \boldsymbol{R}_{2}:=A^{2} \boldsymbol{E}-B_{i} B_{j} \boldsymbol{I}-C^{2} \boldsymbol{E}^{-1}, \\
& \boldsymbol{R}_{3}:=A \boldsymbol{E} \boldsymbol{R}_{2}+F \boldsymbol{I}, \\
& \boldsymbol{R}_{4}:=A \boldsymbol{E} \boldsymbol{R}_{3}-G \boldsymbol{I} .
\end{aligned}
$$

Here the notation used is in agreement with (3.3), and

(3.8) $\quad F:=C^{(1)}\left(B_{i} B_{i}^{(1)}+B_{j} B_{j}^{(1)}\right), \quad G:=C^{(1)} C^{(2)}\left(B_{i} B_{j}^{(2)}+B_{j} B_{i}^{(2)}\right)$.

Pro of. We have

$$
\begin{aligned}
& A P_{i}^{(1)}+B_{i} P_{i}^{(0)}+C P_{i}^{(-1)}=0, \\
& A P_{j}^{(1)}+B_{j} P_{j}^{(0)}+C P_{j}^{(-1)}=0 .
\end{aligned}
$$

Multiplying (3.9) by $A P_{j}^{(1)}$, and making use of (3.10), we obtain

$$
\boldsymbol{R}_{2} w=C\left[B_{i} P_{i}^{(0)} P_{j}^{(-1)}+B_{j} P_{j}^{(0)} P_{i}^{(-1)}\right]
$$

with the operator $\boldsymbol{R}_{2}$ given by (3.5). 
Applying the operator $A \boldsymbol{E}$ to both sides of Eq. (3.11), and making use of (3.9) and (3.10), we get

$$
\boldsymbol{R}_{3} w=-C C^{(1)}\left[B_{j}^{(1)} P_{i}^{(0)} P_{j}^{(-1)}+B_{i}^{(1)} P_{j}^{(0)} P_{i}^{(-1)}\right]
$$

with the operator $\boldsymbol{R}_{3}$ given by (3.6).

Repeating the above process for Eq. (3.12), we obtain

$$
\boldsymbol{R}_{4} w=C C^{(1)} C^{(2)}\left[B_{i}^{(2)} P_{i}^{(0)} P_{j}^{(-1)}+B_{j}^{(2)} P_{j}^{(0)} P_{i}^{(-1)}\right],
$$

where the operator $\boldsymbol{R}_{4}$ is given by (3.7).

Eqs. (3.11), (3.12) and (3.13) imply

$$
\left|\begin{array}{ccc}
\boldsymbol{R}_{2} w & B_{i} & B_{j} \\
\boldsymbol{R}_{3} w & -C^{(1)} B_{j}^{(1)} & -C^{(1)} B_{i}^{(1)} \\
\boldsymbol{R}_{4} w & C^{(1)} C^{(2)} B_{i}^{(2)} & C^{(1)} C^{(2)} B_{j}^{(2)}
\end{array}\right|=0
$$

as $B_{j}^{(m)}=\left(\lambda_{j}-\lambda_{i}\right)+B_{i}^{(m)}$ (cf. (3.2)), this is equivalent to (3.4).

Corollary 3.2. An equivalent form of the difference equation (3.4) is

$$
\left(\boldsymbol{S}_{2} \boldsymbol{R}_{2}+\boldsymbol{T}_{1}\right) w=0
$$

where the difference operator $\boldsymbol{R}_{2}$ is given in (3.5), and

$$
\begin{aligned}
& \boldsymbol{S}_{2}:=A A^{(1)} W_{1} \boldsymbol{E}^{2}+A C^{(2)} W_{2} \boldsymbol{E}+C^{(1)} C^{(2)} W_{3} \boldsymbol{I}, \\
& \boldsymbol{T}_{1}:=A F^{(1)} W_{1} \boldsymbol{E}+H \boldsymbol{I} .
\end{aligned}
$$

Here we use the notation

$$
\begin{aligned}
W_{1} & :=B_{i}+B_{j}^{(1)}, \quad W_{2}:=B_{i}^{(2)}-B_{i}, \quad W_{3}:=-B_{i}^{(2)}-B_{j}^{(1)}, \\
H & :=C^{(2)} F W_{2}-G W_{1} .
\end{aligned}
$$

Proof. Expanding the determinant (3.4) with respect to the first column, we obtain

$$
\boldsymbol{Q}_{4}=C^{(1)} C^{(2)} W_{3} \boldsymbol{R}_{2}+C^{(2)} W_{2} \boldsymbol{R}_{3}+W_{1} \boldsymbol{R}_{4} .
$$

On using (3.6) and (3.7), and rearranging terms, the result follows.

If $i=j$, a slight modification of the argument given in the proof of Theorem 3.1 and Corollary 3.2 leads to the following result.

Theorem 3.3. The square $w:=P_{i}^{2}(i \in \mathbb{N})$ obeys the third-order difference equation

$$
\boldsymbol{Q}_{3} w \equiv\left|\begin{array}{cc}
C^{(1)} \boldsymbol{R}_{2} w & B_{i} \\
\boldsymbol{R}_{3} w & -B_{i}^{(1)}
\end{array}\right|=0
$$


notation used being that of (3.5) and (3.6) (with $i=j$ ). An equivalent form of this equation is

$$
\left(\boldsymbol{S}_{1} \boldsymbol{R}_{2}+\boldsymbol{T}_{0}\right) w=0
$$

where

$$
\begin{aligned}
\boldsymbol{R}_{2} & :=A^{2} \boldsymbol{E}-B_{i}^{2} \boldsymbol{I}-C^{2} \boldsymbol{E}^{-1}, \\
\boldsymbol{S}_{1} & :=A B_{i} \boldsymbol{E}+B_{i}^{(1)} C^{(1)} \boldsymbol{I}, \\
\boldsymbol{T}_{0} & :=2 B_{i}^{2} B_{i}^{(1)} C^{(1)} \boldsymbol{I} .
\end{aligned}
$$

In the next theorem, we give an alternative derivation of the fourth-order difference equation for $P_{i} P_{j}$. It should be stressed that this time the case of $i=j$ is not excluded.

TheOREm 3.4. For any $i, j \geq 0$, the product $w=P_{i} P_{j}$ satisfies the fourth-order difference equation

$$
\widetilde{\boldsymbol{Q}}_{4} w=0
$$

with

$$
\widetilde{\boldsymbol{Q}}_{4}=\boldsymbol{N}_{2} \boldsymbol{M}_{2}-\lambda_{i} \lambda_{j} \boldsymbol{K}_{2},
$$

where

$$
\begin{aligned}
& \boldsymbol{N}_{2}:=\alpha(x)\left[\varphi_{0}(x) \boldsymbol{V}+\varphi_{1}(x) \boldsymbol{I}\right]-\beta(x)\left[\psi_{0}(x) \boldsymbol{U}+\psi_{1}(x) \boldsymbol{I}\right] \\
& \boldsymbol{M}_{2}:=\boldsymbol{L}+\left(\lambda_{i}+\lambda_{j}\right) \boldsymbol{I}
\end{aligned}
$$

and where

$$
\begin{aligned}
\alpha & :=A^{(-1)}\left[B_{i}+B_{j}+\nabla(A+C)\right], & \psi_{0} & :=C^{(-1)}, \\
\beta & :=C^{(1)}\left[B_{i}+B_{j}-\Delta(A+C)\right], & \psi_{1} & :=-A\left[A^{(-1)}+C^{(-1)}\right]-\frac{1}{2} \alpha, \\
\varphi_{0} & :=A^{(1)} & \eta & :=C+C^{(1)}, \\
\varphi_{1} & :=\left[A^{(1)}+C^{(1)}\right] C+\frac{1}{2} \beta, & \vartheta & :=-A-A^{(-1)} .
\end{aligned}
$$

Proof. Let $w:=P_{i} P_{j}$. Using Leibniz' rules

$$
\left\{\begin{array}{l}
\boldsymbol{\Delta}(f g)=f \boldsymbol{\Delta} g+g^{(1)} \boldsymbol{\Delta} f \\
\boldsymbol{\nabla}(f g)=f \boldsymbol{\nabla} g+g^{(-1)} \boldsymbol{\nabla} f,
\end{array}\right.
$$

and the difference equations satisfied by $P_{i}$ and $P_{j}$ (cf. (2.3)), it can be checked that

$$
\boldsymbol{M}_{2} w=A \boldsymbol{\Delta} P_{i} \boldsymbol{\Delta} P_{j}+C \boldsymbol{\nabla} P_{i} \boldsymbol{\nabla} P_{j},
$$

where we use the notation (3.26) and (3.2). Using this result and the identity $C\left[\lambda_{i} P_{i} \boldsymbol{\nabla} P_{j}+\lambda_{j} P_{j} \nabla P_{i}\right]-A\left[\lambda_{i} P_{i} \Delta P_{j}+\lambda_{j} P_{j} \Delta P_{i}\right]=2 \lambda_{i} \lambda_{j} w$, 
we obtain

$$
\begin{aligned}
A \boldsymbol{\Delta}\left(A \boldsymbol{M}_{2} w\right)= & -\left(A^{2}+C C^{(1)}\right) \boldsymbol{M}_{2} w \\
& +\lambda_{i} \lambda_{j}\left[A \boldsymbol{\Delta}+\left(A+C^{(1)}\right) \boldsymbol{I}\right] w-\beta A \boldsymbol{\Delta} P_{i} \boldsymbol{\Delta} P_{j}, \\
C \boldsymbol{\nabla}\left(C \boldsymbol{M}_{2} w\right)= & \left(C^{2}+A A^{(-1)}\right) \boldsymbol{M}_{2} w \\
& +\lambda_{i} \lambda_{j}\left[C \boldsymbol{\nabla}-\left(C+A^{(-1)}\right) \boldsymbol{I}\right] w+\alpha C \boldsymbol{\nabla} P_{i} \boldsymbol{\nabla} P_{j} .
\end{aligned}
$$

On subtracting the equations (3.31) and (3.32), multiplied by $\alpha$ and $\beta$, respectively, and making use of (3.29) and (3.30), the result follows.

3.2. Recurrence relation for the linearization coefficients. For some technical reasons, it is easier to construct a recurrence

$$
\mathcal{L} s_{k}^{i j} \equiv \sum_{h=0}^{r} A_{h}(k) s_{k+h}^{i j}=0
$$

for

$$
s_{k}^{i j}:=\sum_{x=0}^{B-1} \varrho(x) P_{i}(x) P_{j}(x) P_{k}(x),
$$

obviously equivalent to (1.2), in view of

$$
s_{k}^{i j}=h_{k} c_{k}^{i j} .
$$

Now, we prove

THEOREM 3.5. For arbitrary $i, j \geq 0$, the recurrence relation

$$
\mathcal{L} s_{k}^{i j}=0
$$

holds, where

$(3.37) \quad \mathcal{L}:=\alpha(X)\left\{\left[\varphi_{1}(\mathcal{X})-\varphi_{0}(\mathcal{X}) \widetilde{\mathcal{D}}\right]\left(\omega_{k} \mathcal{J}\right)-\lambda_{i} \lambda_{j}[\eta(\mathcal{X})-\widetilde{\mathcal{D}}]\right\}$

$$
-\beta(\mathcal{X})\left\{\left[\psi_{1}(\mathcal{X})-\psi_{0}(\mathcal{X}) \mathcal{D}\right]\left(\omega_{k} \mathcal{J}\right)-\lambda_{i} \lambda_{j}[\vartheta(X)-\mathcal{D}]\right\},
$$

with $\omega_{k}:=\lambda_{i}+\lambda_{j}-\lambda_{k}$, notation being that of (2.10)-(2.12), (3.28).

Proof. Let $w:=P_{i} P_{j}$. Obviously,

$$
s_{k}^{i j}=b_{k}[w], \quad c_{k}^{i j}=a_{k}[w] .
$$

By virtue of Theorem 3.4,

$$
b_{k}\left[\widetilde{\boldsymbol{Q}}_{4} w\right]=0 .
$$

It suffices to show that the identity

$$
b_{k}\left[\widetilde{\boldsymbol{Q}}_{4} w\right]=\mathcal{L} b_{k}[w]
$$

holds. Now, observe that by Lemma 2.1, we have the following identities:

$$
\begin{aligned}
b_{k}\left[\boldsymbol{N}_{2} z\right] & =\left\{\alpha(X)\left[\varphi_{1}(X)-\varphi_{0}(X) \widetilde{\mathcal{D}}\right]-\beta(X)\left[\psi_{1}(X)-\psi_{0}(X) \mathcal{D}\right]\right\} b_{k}[z], \\
b_{k}\left[\boldsymbol{M}_{2} w\right] & =\left(\lambda_{i}+\lambda_{j}-\lambda_{k}\right) b_{k}[w],
\end{aligned}
$$




$$
b_{k}\left[\boldsymbol{K}_{2} w\right]=\{\alpha(X)[\eta(X)-\widetilde{\mathcal{D}}]-\beta(X)[\vartheta(X)-\mathcal{D}]\} b_{k}[w] .
$$

From (3.24)-(3.27), applying again Lemma 2.1, we obtain (3.38).

Obviously, we have the following.

COROLLARY 3.6. The linearization coefficients $c_{k}^{i j}$ in (1.1) obey the recurrence relation

$$
\mathcal{L}^{*} c_{k}^{i j}=0
$$

with $\mathcal{L}^{*}:=\mathcal{L}\left(h_{k} \mathcal{J}\right), \mathcal{L}$ being the difference operator given in (3.37).

EXAmPle 3.7. The coefficients $\left\{c_{k}^{i j}\right\}$ in

$$
C_{i}(x ; a) C_{j}(x ; a)=\sum_{k=|i-j|}^{i+j} c_{k}^{i j} C_{k}(x ; a) \quad\left(x \in \mathbb{N}_{0}\right),
$$

where $C_{m}(x ; a)$ is the $m$ th monic Charlier polynomial (see Appendix, Table 1), satisfy the sixth-order recurrence relation

$$
\sum_{h=-3}^{3} B_{h}(k) c_{k+h}^{i j}=0 \quad(|i-j|+3 \leq k \leq i+j+2),
$$

with

$$
\begin{aligned}
B_{-3}(k)= & 2(k-s-3), \\
B_{-2}(k)= & (k-s-2)(6 k+8 a-s+1)+2 i j, \\
B_{-1}(k)= & (k-s-1)\left[6 k^{2}+2(11 a-s+4) k-s+1+2 a(4 a+7)\right] \\
& +i j(4 k+12 a-s+5), \\
B_{0}(k)= & (k-s)\left\{2 k^{3}+(7-s+20 a) k^{2}\right. \\
& \left.+2\left(11 a^{2}+23 a+3-s\right) k+2 a^{2}(3 s+13)-a\left(s^{2}-25\right)\right\} \\
& +i j\left[2 k^{2}+(7-s+22 a) k+6(a+1)(4 a+1)-2 s(2 a+1)\right], \\
B_{1}(k)= & a(k-s+1)\left\{6 k^{3}+10(2 a+3) k^{2}+\left[49-s^{2}+a(9 s+67)+4 a^{2}\right] k\right. \\
& \left.+4(s+1) a^{2}+\left(58+15 s-s^{2}\right) a-2 s^{2}+26\right\} \\
& +2 a i j\left[5 k^{2}+2(9+10 a-s) k+2(a+2)(4 a-s+8)-16\right], \\
B_{2}(k)= & a^{2}(k+2)\{(k-s+2)[3(k+3)(2 k+3)+3(s+2 a)(k+1) \\
& -(s-6 a-4)(s+1)]+4 i j(4 k-s+6 a+8)\}, \\
B_{3}(k)= & 2 a^{3}(k+2)_{2}(k+i-j+3)(k-i+j+3),
\end{aligned}
$$

where $s:=i+j$. The initial conditions are $c_{i+j}^{i j}=1$, and $c_{m}^{i j}=0$ for $m>i+j$. Actual forms for $B_{h}$ 's were obtained using the computer algebra system MAPLE [3]. 
EXAMPLE 3.8. The coefficients $\left\{c_{k}^{i j}\right\}$ in

$$
K_{i}(x ; 1 / 2, N) K_{j}(x ; 1 / 2, N)=\sum_{k=|i-j|}^{i+j} c_{k}^{i j} K_{k}(x ; 1 / 2, N) \quad(0 \leq x \leq N),
$$

where $K_{m}(x ; 1 / 2, N)$ is a special case of the $m$ th monic Krawtchouk polynomial (see Appendix, Table 2), satisfy the three-term recurrence relation $16(k-s-2)(2 N-s-k+2) c_{k-2}^{i j}$

$$
\begin{aligned}
& +4\left[\left(k^{2}-d^{2}\right)(k-N-2)_{2}-(k+1)_{2}(k-s)(2 N-s-k)\right] c_{k}^{i j} \\
& -(k+1)_{2}\left[(k+2)^{2}-d\right](k-N)_{2} c_{k+2}^{i j}=0 \quad(|i-j|+2 \leq k \leq i+j+1),
\end{aligned}
$$

where $s:=i+j$, and $d:=i-j$. The starting values are $c_{i+j}^{i j}=1$, and $c_{m}^{i j}=0$ for $m>i+j$. This result agrees with the explicit form given in [2].

Acknowledgments. A part of this work was done during a visit of one of the authors (S. L.) at the Université des Sciences et Technologies de Lille. He is very indebted to Professor Claude Brezinski, Directeur du Laboratoire d'Analyse Numérique et d'Optimisation, and Professor Jeannette Van Iseghem for the kind invitation and their warm hospitality.

\section{Appendix}

T A B L E 1

Data for the monic Charlier and Hahn polynomials

\begin{tabular}{ccc}
\hline & ChARLIER & HAHN \\
& $\begin{array}{c}C_{k}(x ; a) \\
(a>0)\end{array}$ & $\begin{array}{c}Q_{k}(x ; \alpha, \beta, N) \\
\left(\alpha, \beta>-1, N \in \mathbb{Z}^{+}\right)\end{array}$ \\
\hline$\sigma$ & $x$ & $x(N+\alpha-x)$ \\
$\tau$ & $a-x$ & $(\beta+1)(N-1)-(\gamma+1) x$ \\
$\lambda_{k}$ & $k$ & $k(k+\gamma)$ \\
$X$ & $a k \mathcal{E}^{-1}+(k+a) \mathcal{J}+\mathcal{E}$ & $\frac{k(N-k)(k+\alpha)(k+\beta)(k+\gamma-1)(k+\gamma+N-1)}{(2 k+\gamma-2)_{2}(2 k+\gamma-1)_{2}} \mathcal{E}^{-1}$ \\
& & $+\left\{\frac{\alpha-\beta+2 N-2}{4}+\frac{\left(\beta^{2}-\alpha^{2}\right)(\gamma+2 N-1)}{4(2 k+\gamma-1)(2 k+\gamma+1)}\right\} \mathcal{J}+\mathcal{E}$ \\
& & $\frac{k(k+\alpha)(k+\beta)(k+\gamma-1)_{2}(N-k)(k+\gamma+N-1)}{(2 k+\gamma-2)_{2}(2 k+\gamma-1)_{2}} \mathcal{E}^{-1}$ \\
$\mathcal{D}$ & $a k \mathcal{E}^{-1}$ & $-\frac{k(k+\gamma)[2 k(k+\gamma)+(\gamma-\alpha)(\gamma-1)-N(\alpha-\beta)]}{(2 k+\gamma-1)(2 k+\gamma+1)} \mathcal{J}-k \mathcal{E}$ \\
& & $\frac{k ! \Gamma(k+\alpha+1) \Gamma(k+\beta+1)(2 k+\gamma+1)_{N-k-1}}{(k+\gamma)_{k}(N-k-1) !}$ \\
$h_{k}$ & $k ! a^{k}$ &
\end{tabular}

Note: $\gamma:=\alpha+\beta+1$. 
T A B LE 2

Data for the monic Meixner and Krawtchouk polynomials

\begin{tabular}{ccc}
\hline & MEIXNER & KRAWTCHOUK \\
& $M_{k}(x ; \beta, c)$ & $K_{k}(x ; p, N)$ \\
& $(\beta>0, c \in(0,1))$ & $\left(p \in(0,1), N \in \mathbb{Z}^{+}\right)$ \\
\hline$\sigma$ & $x$ & $x$ \\
$\tau$ & $\beta c+(c-1) x$ & $(1-p)^{-1}(N p-x)$ \\
$\lambda_{k}$ & $(1-c) k$ & $(1-p)^{-1} k$ \\
$X$ & $\frac{c k(k+\beta-1)}{(1-c)^{2}} \mathcal{E}^{-1}$ & $p(1-p) k(N-k+1) \mathcal{E}^{-1}$ \\
& $+\frac{[(c+1) k+\beta c]}{1-c} \mathcal{J}+\mathcal{E}$ & $+[k+p(N-2 k)] \mathcal{J}+\mathcal{E}$ \\
$\mathcal{D}$ & $\frac{c k(1-\beta-k)}{c-1} \mathcal{E}^{-1}+c k \mathcal{J}$ & $p k(1+N-k) \mathcal{E}^{-1}-p(1-p)^{-1} k \mathcal{J}$ \\
$h_{k}$ & $\frac{k !(\beta)_{k} c^{k}}{(1-c)^{\beta+2 k}}$ & $\frac{N ! k !}{(N-k) !} p^{k}(1-p)^{k}$ \\
\hline
\end{tabular}

\section{References}

[1] R. Askey, Orthogonal Polynomials and Special Functions, Regional Conf. Ser. Appl. Math. 21, SIAM, Philadelphia, 1975.

[2] R. Askey and G. Gasper, Convolution structures for Laguerre polynomials, J. Anal. Math. 31 (1977), 48-68.

[3] B. W. Char, K. O. Geddes, G. H. Gonnet, B. L. Leong, M. B. Monagan and S. M. Watt, Maple V Language Reference Manual, Springer, New York, 1991.

[4] T. S. Chihara, An Introduction to Orthogonal Polynomials, Gordon and Breach, New York, 1978.

[5] A. G. Garcia, F. Marcellán and L. Salto, A distributional study of discrete classical orthogonal polynomials, J. Comput. Appl. Math. 57 (1995), 147-162.

[6] R. Koekoek and R. F. Swart touw, The Askey scheme of hypergeometric orthogonal polynomials and its q-analogue, Fac. Techn. Math. Informatics, Delft Univ. of Technology, Rep. 94-05, Delft, 1994.

[7] J. Letessier, A. Ronveaux and G. Valent, Fourth order difference equation for the associated Meixner and Charlier polynomials, J. Comput. Appl. Math. 71 (1996), 331-341.

[8] S. Lewanowicz, Recurrence relations for the connection coefficients of orthogonal polynomials of a discrete variable, ibid. 76 (1996), 213-229.

[9] A. F. Nikiforov, S. K. Suslov and V. B. Uvarov, Classical Orthogonal Polynomials of a Discrete Variable, Springer, Berlin, 1991.

[10] A. Ronveaux, S. Belmehdi, E. Godoy and A. Zarzo, Recurrence relations approach for connection coefficients-Applications to classical discrete orthogonal polynomials, in: Symmetries and Integrability of Difference Equations, D. Levi, 
L. Vinet and P. Winternitz (eds.), Centre de Recherches Mathématiques, CRM Proc. and Lecture Notes Ser. 9, Amer. Math. Soc., Providence, 1996, 321-337.

Saïd Belmehdi

UFR de Mathématiques

Université des Sciences et Technologies de Lille

59655 Villeneuve d'Ascq, France

E-mail: belmehdi@ano.univ-lille1.fr

André Ronveaux

Laboratoire de Physique Mathématique

Facultés Universitaires N.-D. de la Paix

B-5000 Namur, Belgium

E-mail: Andre.Ronveaux@fundp.ac.be
Stanisław Lewanowicz Institute of Computer Science

University of Wrocław

51-151 Wrocław, Poland

E-mail: Stanislaw.Lewanowicz@ii.uni.wroc.pl

Received on 7.11 .1996 